\title{
Pharmacy Transition Care Services during Mass Gathering Hajj in Saudi Arabia
}

Yousef Ahmed Alomi* iD, Critical Care Clinical Pharmacists, TPN Clinical Pharmacist, Freelancer Business Planner, Content Editor and Data Analyst, Riyadh, Saudi Arabia.

Nezar Adnan Yahya Khayat, Bsc. Pharm Medication Safety Officer, Pharmaceutical Care Administration, Makkah Medical Affairs Region, Makkah, Saudi Arabia.

Yasir Abdulraheem Bamagaus, Bsc. Pharm Regional General Manager, Pharmaceutical Care Administration, Makkah Medical Affairs Region, Makkah, Saudi Arabia.

Naif Olaythah Alharbi, Diploma. Pharm

Staff Pharmacist, Pharmaceutical Care Administration, Makkah Medical Affairs Region, Makkah, Saudi Arabia.

Fareed Ali Alharpi, Bsc. Nursing

Nursing staff, Makkah Medical Affairs Region,

Makkah, Saudi Arabia.

Ahmad M. AlKharoby, MD

Assistant General Manager for assistant medical affiars, Makkah Medical Affairs Region, Makkah, Saudi Arabia.

Wael H. Motair, MD

General Manager, Makkah Medical Affairs

Region, Makkah, Saudi Arabia.

\section{Correspondence:}

Dr. Yousef Ahmed Alomi, (BSc. Pharm, MSc. Clin Pharm, BCPS, BCNSP, DiBA, CDE)

Critical Care Clinical Pharmacists, TPN Clinical Pharmacist, Freelancer Business Planner, Content Editor and Data Analyst, P.O. BOX 100, Riyadh-11392, Riyadh, Saudi Arabia.

Phone no: +966504417712

E-mail:yalomi@gmail.com

Received: 20-09-2019;

Accepted: 27-11-2019

Copyright: (c) the author(s), publisher and licensee International Journal of Pharmacology and Clinical Sciences. This is an open-access article distributed under the terms of the Creative Commons Attribution Non-Commercial License, which permits unrestricted non-commercial use, distribution, and reproduction in any medium, provided the original work is properly cited.

This is an open access article distributed under the terms of the Creative Commons

Access this article online

\begin{tabular}{|c|c|}
\hline & www.ijpcs.net \\
\hline & DOI: \\
\hline
\end{tabular}

\begin{abstract}
Objective: To declare the pharmacy transition care services during the mass gathering hajj period as new initiatives in the Kingdom of Saudi Arabia. Methods: It is a new initiative project drove by national mass gathering pharmaceutical programs. The projects drove the mass gathering medicine guidelines and the international business model, pharmacy project guidelines project management institution guidelines of a new project. The initiative project is written through project management professionals and consisted of several parts, including the initial phase, the planning phase, the execution phase and the monitoring and controlling phase. Results: The mass gathering pharmacy transition care services with a defined vision, mission and goals. The services had multiple benefits, including clinical and economical on pilgrims, as demonstrated in the review. The extension of the project assured by risk management elements description. In addition, the monitoring and controlling of the services as illustrated. The conversion of operation project, though closing project stage, demonstrated in the analysis. Conclusion: The pharmacy transition care services during the mass gathering is a new initiative, part of the mass gathering medicine. Various pharmacy transition care services can be started during mass gathering; it is highly recommended to implement in Saudi Arabia.
\end{abstract}

Keywords: Mass gathering, Hajj, Pharmacy, Transition Care, Services, Initiative, Saudi Arabia.

\section{INTRODUCTION}

There are several healthcare services offers to all patients, including pilgrims during mass gathering hajj period in the kingdom of Saudi Arabia. ${ }^{[1]}$ The patients can receive healthcare from the hospital or primary healthcare centers. In-hospital care, multiple services received daily; that is included emergency, ambulatory care, critical care and acute care services. ${ }^{[1]}$ The patients or pilgrims can be transferred from service to another according to the patient's conditions. The healthcare system should be continuous with high advance care. There is a system when patients or pilgrims during mass gathering hajj transfers from one service to another called transition care services. ${ }^{[2-4]}$ Besides, the patients can transfer from inside the hospitals to outside another hospital or from primary healthcare centers to hospitals within the local city or around any city inside or outside Saudi Arabia. The transition care system, including pharmacy transition care services, was implemented during mass gathering hajj period when the patients wish to continue their hajj activities and transfer from one healthcare unit to another healthcare service inside the holy places. During the mass gathering hajj period, the pilgrims should stay in the holy places to complete hajj performance and each activity should be done within specific days. As a result, pharmacy transition care services play a significant role during the mass gathering hajj period. The involvement of pharmacists in the transition care services will improve pharmaceutical care provided to the patients and prevent drug misadventures. ${ }^{[5]}$ Various studies showed an excellent impact on pharmacists during transition care services. ${ }^{[5-8]}$ Based on the knowledge of the authors, they are not aware of any investigations or review discussed the pharmacy transition care services during mass gathering hajj period locally or Gulf and the Middle East or the rest of the world countries. ${ }^{[9,10]}$ The aim of the project is to demonstrate the pharmacy transition care services during a mass gathering hajj period in the kingdom of Saudi Arabia.

\section{Method of the Project}

It is a new initiative project drove by national mass gathering pharmaceutical programs. The task force team of mass gathering hajj pharmacy projects formulated and consisted of from an author's expert in the mass gathering hajj. The committee unitized and drove the mass gathering medicine guidelines and from the textbook and international literature about mass gathering medications, safety written by utilizing the international business model, pharmacy project guidelines project management institution guidelines of a new project. ${ }^{[11-14]}$ The mass gathering medication safety project written through project management professionals and consisted of several parts, including the initial phase, the planning phase, the execution phase and the monitoring and controlling phase.

Initiative Phase 


\section{Assessment Needs}

Transitional care is the vital system in the hospital care and pharmacy services, had a significant role through improved patient care, prevent drug-related problems, prevent an additional unnecessary economic burden on the healthcare system. ${ }^{[5-8]}$ The pharmacy transition care involved over several services with new admissions, patients transferred from any other healthcare services, or patients discharged to home care or other healthcare organizations. Some healthcare organizations implemented those services. ${ }^{[9,10]}$ However, not all healthcare institutions implemented the services, including transfers from one institution to another. Several hospitals involved during mass gathering hajj period with the medication reconciliation system. The pharmacy transition services with complete service not fully implemented during the mass gathering hajj period.

\section{Market Analysis}

In the healthcare practice, there are several types of patient transfers, daily from one hospital to another and from one general hospital units to a specialized hospital department. However, the exact system or policy and pro $\neg$ cedures not existed involving the pharmacy services at all Ministry of Health hospitals and especially during mass gathering hajj period. There are policies and procedures of transition care services during mass gathering hajj from hospitals located in the holy Makkah to the holy place, but the complete system related to medications and pharmacist jobs not included.

\section{SWOT Analysis}

Each new project should analyze through several methods to declare the expected positive or negative outcome of the project. One of the popular tools used was SWOT. The first point of the analysis was the strength points of the project that's including preventing medication errors during the transition, prevent drug-related problems after the transition and reduce the cost of medication errors, more compliance with the medications. The weak points of the project need training, need policy and procedures and financial support. The opportunity points of the project, where the project fit with CBAHI standards and international accreditation by the body; ${ }^{[15,16]}$ it fills with $\mathrm{MOH}$ healthcare strategic plan and new vision 2030. ${ }^{[17]}$ The thread points were the changing of the $\mathrm{MOH}$ plan, no grant support for education and changing transition services of the $\mathrm{MOH}$ institutions.
Planning Phase

\section{Scope of the Project}

The pharmacy Transition care services cover any patients transfer from one unit to another department at the same hospital or transfer patients from hospital to another or transfer patients from hospital to the holy places during mass gathering hajj period.

\section{Vision, Mission and Goals \\ Vision}

The best pharmaceutical care services during the patient's transition or transfer of services to another at higher or lower healthcare levels.

\section{Mission}

To provide pharmacy transition care services to all patients during regular or mass gathering hajj days.

\section{Goals}

1. To prevent drug-related problems during hospital transition care services

2. To prevent medications discrepancy during transition care services

3. To avoid additional unnecessary financial burden on health care services

4. To follow up and monitory the patient's condition during transition care services

5. To provide patients medications education during transition care services

\section{Project Description}

$\checkmark$ When the hospital transition care committee agreed on the quantity and quality of the patient's candidate to complete his hajj performance through hospital transition care services, the patient's list had been sent to nursing units.

$\checkmark$ The transition care team contained physician, nurse and clinical pharmacist or trained pharmacist make around to all pilgrim patients.

$\checkmark$ The team reviews all the cases candidates for transition to the holy places to continue their hajj performance.

$\checkmark$ When the team agrees with the patients for transition care services, the team will review all things needs for an instant; artificial equipment, medications, nutrition and related issues.

$\checkmark$ The clinical pharmacist among the team reviews the medications for pharmacy transition care that's including Medications therapeutic interchange system from parental to oral dosage and appropriate for transition care.

$\checkmark$ The physician among the transition care team approved the transition medications and wrote the transition care prescription through manually or electronically. $\checkmark$ The clinical pharmacist will document the therapeutic exchange approved through the pharmacist intervention system.

$\checkmark$ All the transition care prescription sends to the inpatient pharmacy though transition care nurses.

$\checkmark$ The pharmacist review medication history before and after patient's transition to holy places through the medications reconciliation system.

$\checkmark$ The pharmacist fills the form of a reconciliation system. If there are any discrepancies will be recognized.

$\checkmark$ The pharmacist prepares and fills special trolly and dispense the patient's medications though unit dose drug distribution system and ready packaging ready to administer for $24 \mathrm{hrs}$.

$\checkmark$ If the hospital had an automated dispensing cabinet, the pharmacist, after medication reconciliation, sent authorization to the automated cabinet to dispense the medications for $24 \mathrm{hrs}$.

$\checkmark$ All transition nurses receive the medications from the pharmacy and prepare it for administration through medications portable cabinet.

$\checkmark$ All transition nurses take all medications potable to the particular transition care ambulances or buses and administer to transition patients consequently.

$\checkmark$ Once all transition care ambulance or buses return the patients to their hospital, the transition transfer the patients to their beds.

$\checkmark$ The hospital nurses receive the transition of patients from transition nurses, including their medications.

$\checkmark$ The transition care team, including a physician, nurse and clinical pharmacist or trained pharmacist, will make around to all patients who came from holy places transition care services.

$\checkmark$ The team will analyze the cases, artificial equipment, medications, nutrition and related issues. The team decides which shall continue or discontinue.

$\checkmark$ The clinical pharmacist will review the medications, whether it will continue the dosage of the medications forms or switch to the suitable therapeutic interchange.

$\checkmark$ The transition care physician will write the medications manually or electronically and nurses send the prescription to the pharmacy.

$\checkmark$ The hospital nurses send the potable trolley with all medications, including a new prescription to the pharmacy.

$\checkmark$ The pharmacist receives portable medications, trolley and prescriptions. The pharmacist review medications history after patient's transition to holy places and cur- 
rent situation through medications reconciliation system.

$\checkmark$ The pharmacist fills the form of a reconciliation system. If there are any inconsistencies, it will be documented.

$\checkmark$ The pharmacist prepares and dispenses the appropriate medications to the patients through a trolley or automated dispensing cabinet.

\section{Plan Schedule Management}

The project should be separated into several steps during the implementation. The pharmacy transfer care services started in hospital wards or units. Then revision of the procedure should be done and correct the mistake accordingly. The transition care services can be expended to another hospital with revision. The patient's evolution to holy places should be underway and revised the system and correct the mistakes accordingly.

\section{Cost Management Plan}

The program needs the financial budget for education and training. Some pharmacy devices need to bring it for instant mobile refrigerators, insulin devices storage and an oral syringe. Some education medications material needs to bough for the program.

\section{Executing Phase}

\section{Management Team}

Pharmacy transition care services should be formulated. It consisted of supervisors or acting one of inpatient pharmacy and IV admixture. Besides clinical pharmacy services supervisor. Alternatively, the pharmacist should be a member of the hospital transition care services committee or team.

\section{Education and Training}

This part is crucial before start program implementation. The education and training are highly recommended for the pharmacy transition care services team. Implementation of the services, pharmacists and pharmacy technicians are working with transition care patients, physicians and nursing staff about the new system of pharmacy transition care services.

\section{Monitoring and Controlling Phase Project Total Quality Management}

The pharmacy transition care services need monitoring system tools to measure the success rate of the programs and prevent any mistakes during the implementation phase. Several vital performance indicators should implement with the project, including the number of discrepancies during medication reconciliation, the clinical and economic impact of pharmacy transition care services, type of medications during transition care, a storage system of medications during transition care services.

\section{Risk Management}

In the current project, there are six types of risks: budget risks, scope risks, personal risks, schedule risk, technical risks and quality risks. The project might experience risks such as budget, personnel and quality risks. The project might maybe have a budget risk due to no availability of the budget for education and training for the healthcare staff, including pharmacists and pharmacy Technicians. The project may experience to personnel risks such as shortage of expert staff with a high workload of documentation discrepancies. Besides, the pharmacy staff has not received education or training about the project. The project might be experienced in quality risks due to the non-qualified pharmacist being available and training in the quality pharmacy tools. The project might be exposed to other technical risks such as non-availability on the electronic system of pharmacy transition care with friendly use.

\section{Closing of the Project}

If all steps of pharmacy transition care services implemented at all levels or the location of healthcare services. The project should be continued on regular and routine work regularly. The project should switch from project to the operational preparation system in the pharmacy practice system.

\section{ACKNOWLEDGEMENT}

None.

\section{CONFLICT OF INTEREST}

The authors declare no conflict of interest.

\section{ABBREVIATIONS}

MOH: Ministry of Health; KSA: Kingdom of Saudi Arabia; SWOT: Strengths, Weaknesses, Opportunities and Threats.

\section{REFERENCES}

1. Saudi Ministry of Health. Health Statistical Year Book 2017. Saudi Ministry of Health. 2017.

2. AGS Health Care Systems Committee. American Geriatrics Society (AGS) Position Statement Improving the Quality of Transitional Care for Persons with Complex Care Needs. 2008:2.

3. Hume AL, Kirwin J, Bieber $H L$, et al. Improving care transitions: Current practice and future opportunities for pharmacists. Pharmacotherapy. 2012;32(11):e326-37.

4. American Society of Health-System Pharmacists, American Pharmacists Association. Best Practices from the ASHP-APhA Medication Management in Care Transitions Initiative. 2013. Available from: https://www.ashp.org/-/media/assets/pharmacypractice/resource-centers/quality-improvement/ learn-about-quality-improvement-medicationmanagement-care-transitions.ashx.

5. Ensing HT, Stuijt CCM, DenBemt BJFV, et al. Identifying the optimal role for pharmacists in care transitions: A systematic review. J Manag Care Pharm. 2015;21(8):614-38.

6. Lacy MC, Bryant GA, Herring MS, et al. Implementation and evaluation of a pharmacy-driven transitions of care program for patients discharged from the emergency department. J Am Coll Clin Pharm. 2019;2(1):8-13.

7. Ni W, Colayco D, Hashimoto J, et al. Budget impact analysis of a pharmacist-provided transition of care program. J Manag Care Spec Pharm. 2018;24(2):90-6.

8. Hammad EA, Bale A, Wright DJ, et al. Pharmacy led medicine reconciliation at hospital: A systematic review of effects and costs. Res Soc Adm Pharm. 2017;13(2):300-12.

9. Al-Rashoud I, Al-Ammari M, Al-Jadhey $\mathrm{H}$, et al. Medication discrepancies identified during medication reconciliation among medical patients at a tertiary care hospital. Saudi Pharm J. 2017;25(7):1082-5.

10. Mazhar F, Akram S, Al-Osaimi YA, et al. Medication reconciliation errors in a tertiary care hospital in Saudi Arabia: Admission discrepancies and risk factors. Pharm Pract. 2017;15(1).

11. McDonough R. Writing a Business Plan for a New Pharmacy Service. The Dynamics of Pharmaceutical Care: Enriching Patients' Health. 2010;23.

12. Harris IM, Baker E, Berry TM, et al. Developing a Business-Practice Model for Pharmacy Services in Ambulatory Settings. Pharmacotherapy. 2008;28(2):7e-34e.

13. Sachdev G. Sustainable business models: Systematic approach toward successful ambulatory care pharmacy practice. Am J Heal Pharm. 2014;71(16):1366-74.

14. PMBOK Guide. A guide to the project management body of knowledge. Sixth Edition. Project Management Institute Inc. 2017.

15. Medication management system. Saudi Center Board for Accreditation for Healthcare Institutions (CBAHI). 2016.

16. The Joint Commission. 2016 Comprehensive Accreditation Manuals. Joint Commission Resources. 2016

17. Ministry of Health. National E-Health Strategy - $\mathrm{MOH}$ Initiatives 2030. 2017. Available from: https://www.moh.gov.sa/en/Ministry/nehs/Pages/ vision2030.aspx. 